\title{
Can healthy people benefit from health apps?
}

This Head to Head contains some errors (BMJ 2015;350:h1887, doi:10.1136/bmj.h1887). Firstly, reference 2 incorrectly cites a research paper by Carter MC et al to support a statement in the second paragraph on the efficacy of weight loss plan app Lose It. This reference should have cited this paper: Wharton CM, Johnston CS, Cunningham BK, Sterner D. Dietary self-monitoring, but not dietary quality, improves with use of smartphone app technology in an 8-week weight loss trial. $J$ Nutr Educ Behav 2014;46(5):440-4. http://www.ncbi.nlm.nih. gov/pubmed/25220777
Furthermore, the sentence that reference 2 supports notes that the app Lose It "works just as well as or even better than paper based or website based weight loss plans." In fact, the study on Lose It compared the app against a paper based method and the memo feature on a smartphone (not a website).

Finally, the lists of "deserving" and "undeserving" illnesses are the wrong way round in the penultimate paragraph.

Cite this as: BMJ 2015;350:h2520

๑ $\odot$ BMJ Publishing Group Ltd 2015 